\title{
Assessment of on-line rheology as a control tool for thickened tails
}

\author{
Timothy Kealy Rheology Solutions Pty Ltd, Australia \\ Pradipto Bhattacharjee Rheology Solutions Pty Ltd, Australia \\ Patrick Griffin Rheology Solutions Pty Ltd, Australia
}

\begin{abstract}
The intention of this work was to assess the suitability of the online rheometer (OLR) as a process control tool for measuring coal paste, fed direct from thickener through an OLR. The aim being to predict the OLR performance on-site for process control measuring complex viscosity, G' elastic modulus (the 'solid-like' component of the paste), $G$ " viscous modulus (the 'liquid-like' contribution to flow of the paste) controlled by the local operator. On-site testing was conducted to measure the paste density $\left(\mathrm{kg} / \mathrm{m}^{3}\right)$, and compare with Viscoelastic behaviour of the samples. The instrument and sensors used for the analyses were the Rheology Solutions OLR with a serrated plate and plate sensor to minimise slip. The OLR was installed close to the discharge of the thickener, on a rubber mat to damp vibrations from the plant. Thickener discharged thickened paste, which flowed directly through the OLR from the thickener through a 1" diameter flexible hose. OLR data was compared with incumbent process control technology - on-line density measurement. The results indicate that the complex viscosity measured at low frequency correlates well with the in-line density measurement, whereas the other material functions measured by the OLR can be useful for process control. An example of a possible process control strategy is discussed.
\end{abstract}

\section{Introduction}

Waste management practices and facilities are the most significant economic and environmental liability of the mining and mineral processing operations. While remarkable improvement in disposal techniques have been achieved, inefficiencies still exist in the operation of these facilities. Tailing spills, dam failures, seepage, un-rehabilitated sites, and cases of direct discharge into waterways can result in severe and long-term environmental and social consequences that an leave legacies for thousands of years (Leblanc et al., 2000; Kempton et al., 2010). A key component of these difficulties is excess water in the tailings, and the inconsistency of water content of thickener underflow. Density measurements have so far been the go-to technique for operators for estimating the underflow characteristics. But research results show that the yield stress $\left(\tau_{y}\right)$ and the viscosity $(\eta)$ are the two key parameters that are important (Blight and Bentel, 1983). Unfortunately these quantities do not necessarily have a linear correlation with the solids density $(\rho)$ in the underflow, and difficulties arise when they deviate from design specifications and when significantly larger volumes of water need to be managed. Measurements of the yield stress and the viscosity can be accomplished in the laboratory with expensive instrumentation and are usually time consuming. Therefore, techniques like slump tests (Pashias et al., 1996; Clayton et al., 2003) have been used regularly to provide some indicative measure of the flow properties of the thickened underflow in the field. These test however are reliant on the operators experience and results can vary significantly when the same test is conducted by 
a different operator (Gawu and Fourie, 2004). The variations greatly compromise the efficiency of the design of waste management strategies.

In this work, we look at the underflow of a paste thickener used to thicken the tailings from a mine to the desired underflow solids density to enable easy pumping to the reject conveyer that ultimately sends the tailings for disposal. A schematic of the process studied is shown in Figure 1, with the inset on the bottom left showing an image of the tailings emanating from the thickener and the image on the top right showing the removal of tails as they are discharged on to the conveyor. The tailings from several different feed streams (underground and open-pit) are fed to the thickener, and therefore have different particle size distributions and surface chemistries at different points in time. Therefore, the density of the solids in the underflow changes with time, and both the yield stress and viscosity are expected to vary accordingly. While the density can be measured using process instrumentation to a good degree of accuracy, similar confidence cannot be extended to on-site measurements of the yield stress and viscosity due to reasons mentioned earlier.

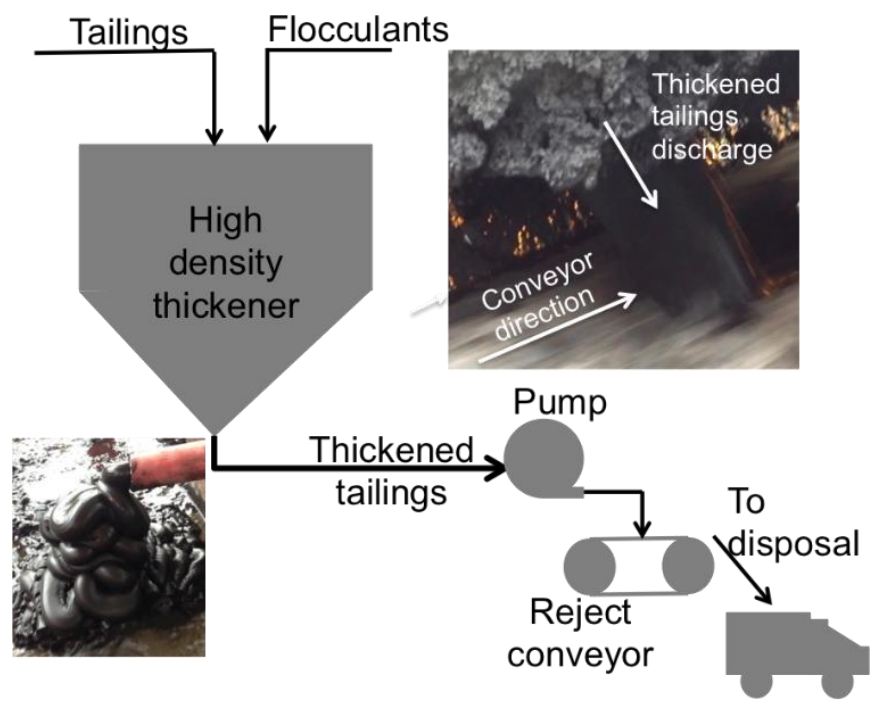

Figure 1 A schematic of the disposal circuit in a typical mine. Images show thickened tailing (bottom left) and conveying of tailings (top right)

In this work we trial a new technology used in OLR Series $1000^{\circ}$ (OLR) that is capable of measuring several rheological flow parameters including the latent elasticity which arises due to the gel-like character of the thickened paste, the viscous property which relates to the flow properties of the material, and a complex viscosity that provides information on the plasticity of the paste. The OLR makes measurements of the rheological properties of a liquid in the frequency domain using oscillatory squeeze flow. During a measurement cycle two parallel plates are brought together trapping a sample of the liquid that is flowing through the pipe. The top plate oscillates over a range of chosen frequencies, and the bottom plate that is connected to a load cell measured the transmitted force. The measured displacement and force profiles then can be used to calculate the storage modulus, representing the elastic component of the liquid $\left(G^{\prime}\right)$, the loss modulus $\left(G^{\prime \prime}\right)$ that represents the viscous component, as a function of frequency $(\omega)$. The details of the physics used for making the measurements and validation of the technique are available elsewhere (Bell et al., 2006; Konigsberg et al., 2013). Once the measurements are completed, the plates separate and the next cycle begins after a desired time interval has passed.

As mentioned previously the OLR measure $\mathrm{G}^{\prime}$ and $\mathrm{G}^{\prime \prime}$ and the complex viscosity $\left(\left|\eta^{*}\right|\right)$ as a function of the angular frequency. Flow properties of tailings are typically discussed in terms of the Herchel Bulkley model (Bird et al., 1977). The most popular expression of this model equation is as follows. 


$$
\tau=\tau_{y}+K \dot{\gamma}^{n}
$$

Where:

$$
\begin{array}{ll}
\tau & =\text { Stress, } \mathrm{Pa} ; \\
\dot{\gamma} & =\text { Shear rate, } \mathrm{s}^{-1} ; \\
\tau_{y} & =\text { Yield stress, } \mathrm{s}^{-1} ; \\
\mathrm{n} & =\text { Plasticity (flow) index, } 0 ; \\
\mathrm{K} & =\text { Consistency index, } \text { Pas }^{\mathrm{n}} ;
\end{array}
$$

When the yield stress is exceeded the second term on the right hand side dominates the rheological response such that $\tau=K \dot{\gamma}^{n}$ and it is possible to write an expression of viscosity as $\eta=K \dot{\gamma}^{-(1-n)}$. For suspensions flocculated with polymeric flocculants, it is possible to make estimates of the $\mathrm{K}$ and $\mathrm{n}$ by fitting a powerlaw trend to the complex viscosity data if the Cox-Merz rule holds (Bird et al., 1977). This is useful since several process parameters, like the Reynolds number depends on $\mathrm{K}$ and $\mathrm{n}$. Therefore, we concentrate mainly on $\eta^{*}$ in this work.

It might be mentioned here that in principle a correlation between can be developed between the complex modulus, $\left|G^{*}\right|$ which is given by $\left|G^{*}\right|=\sqrt{\left(G^{\prime}\right)^{2}+\left(G^{\prime \prime}\right)^{2}}$, and the yield stress $\tau_{y}$, and the correlation can be used to estimate the yield stress from the measure values of $\left|G^{*}\right|$ as has been successfully demonstrated in the literature. It follows that all the relevant parameters of Herschel Bulkley model can be estimated from the measurements made by the OLR. However we do not provide the details here to preserve the brevity of the discussion.

\section{Measurement details and field work}

\subsection{Brief outline of oscillatory squeeze flow for rheometry}

Rheological research into squeeze flow has a rich history and some of the key developments in the area has been reviewed (Engmann et al., 2005). In typical squeeze flow the sample of interest is squeezed between two endplates with the rate of reduction of the separation between the plates determining the rate of strain. The force is typically measured at the bottom plate and the force and the deformation rate data can be combined to extract useful rheological properties of the material. In oscillatory squeeze flow the position of the top plate oscillates with a small amplitude about a mean separation between the plates, which causes the fore on the bottom endplate to also oscillate. It can be shown that amplitude of the oscillatory force depends on the complex viscosity is as follows (Bell et al., 2006; Phan-Thien, 1980).

$$
F_{0}(t)=3 \pi i \omega \epsilon r^{4}\left|\eta^{*}\right| e^{i \omega t}\{1+\alpha\} / 2 h
$$

Where:

$$
\begin{array}{ll}
\omega & =\text { Angular frequency, rad } / \mathrm{s} ; \\
\epsilon \quad & =\text { Displacement amplitude, } \mathrm{m} ; \\
\left|\eta^{*}\right| & =\text { Complex viscosity, Pas; } \\
r \quad & =\text { Radius of top plate, } \mathrm{m} ; \\
t \quad & =\text { Time, } \mathrm{s} ; \\
h & =\text { Separation between plates, } \mathrm{m} ; \\
\alpha & =\text { Frequency dependent correction factor for inertia; }
\end{array}
$$


Thus by setting up $h, r, \epsilon$ and $\omega$, and measuring $\mathrm{F}_{0}$ as a function of time, the $\left|\eta^{*}\right|$ can be measured, and the storage modulus $\mathrm{G}^{\prime}$ and the loss modulus $\mathrm{G}^{\prime \prime}$ that signify the elastic and the viscous properties of the liquid can be calculated once the lag between the deformation and the force is known. This basic scheme is has been broadly applied to laboratory measurements and is also implemented in the OLR here for online measurements of mine tailing. Validation studies (Konigsberg et al., 2013) as well as application to various types of liquids are available in literature (Konigsberg et al., 2014; Wingstrand et al., 2016; Phan-Thien et al., 2000).

\section{$2.2 \quad$ Field trials}

Trials were conducted on-site to assess the suitability of online rheology measurements as a control tool for thickened tails. For conducting these measurements the OLR was placed under the thickener. Thickener discharged thickened paste, which flowed directly through the OLR from the thickener through a 1" diameter flexible hose (with expander/reducer section to 3" on either side of the OLR). Trials were conducted over long (eight hours) and short (forty-five minutes) durations during which the thickener was kept operational. The temperature ranged between 18 and $25^{\circ} \mathrm{C}$ in field trials, and was assessed by measuring the temperature of the stream fed to the OLR.

\section{Results and discussion}

Figure 2(a) shows an example of such a measurement. It can be observed from Figure 2(a) that $\mathrm{G}^{\prime}$ remains almost independent of frequency over the range of $\omega$ used, demonstrating the gel-like nature of the thickened tails. By contrast, $G^{\prime \prime}$ gradually increases with $\omega$, indicating that the viscous component progressively increases with $\omega$. There is a crossover between $G^{\prime}$ and $G^{\prime \prime}$ that occur at a frequency $\omega_{c}=300 \mathrm{rad} / \mathrm{s}$. We note that data at such high frequencies cannot be obtained easily in a typical oscillatory shear flow measurements conducted in the laboratory due to inertial considerations. The use of squeeze flow techniques in the OLR allows the behaviour in this regime to be quantified, and we believe that characterisation of this regime with the degree of clarity shown in Figure 2(a) is rarely available in literature and even less so when the measurements are made online, at the processing site. The complex viscosity

$\eta^{*}(\omega)=\sqrt{\left(G^{\prime} / \omega\right)^{2}+\left(G^{\prime \prime} / \omega\right)^{2}}$, which can be thought to represents the overall resistance to flow is plotted as a function of $\omega$ as a flow curve in Figure 2(b). It can be observed that $\eta^{*} \sim \omega^{-0.7}$ over the range studied. It is possible to fit a power-law trend to the complex viscosity data spanning an angular frequency range of two orders of magnitude available experimentally, and estimate a value of the plasticity parameter ( $\mathrm{n}$ ) and the consistency parameter $(\mathrm{K})$ from the measurements. A typical fit is also shown in Figure $2(\mathrm{~b})$. For the experiment presented $\mathrm{n}=0.2$ and $\mathrm{K}=288 \mathrm{Pas}^{\mathrm{n}}$. When the Cox-Merz rules hold these estimates exactly correspond to the values available from steady shear flow. These results demonstrate the typical set of information available from a single measurement cycle of the OLR. When the OLR is used over a longer period of time for measurements, the acquired data can be used to track the variations of $\mathrm{K}$ and $\mathrm{n}$ with time. In the inset of Figure 2(b) we provide the snapshot of how $K$ and $n$ changes over a period of forty-five minutes of operations of a high-density thickener at a mine site. 


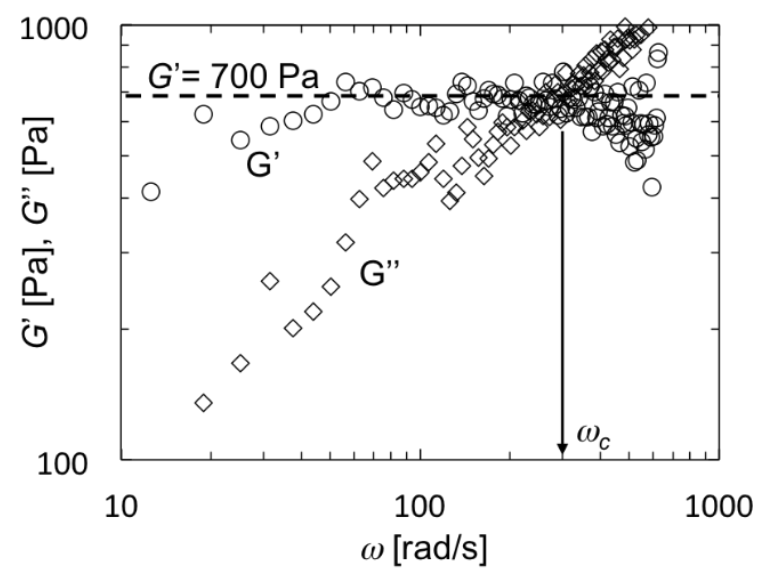

(a)

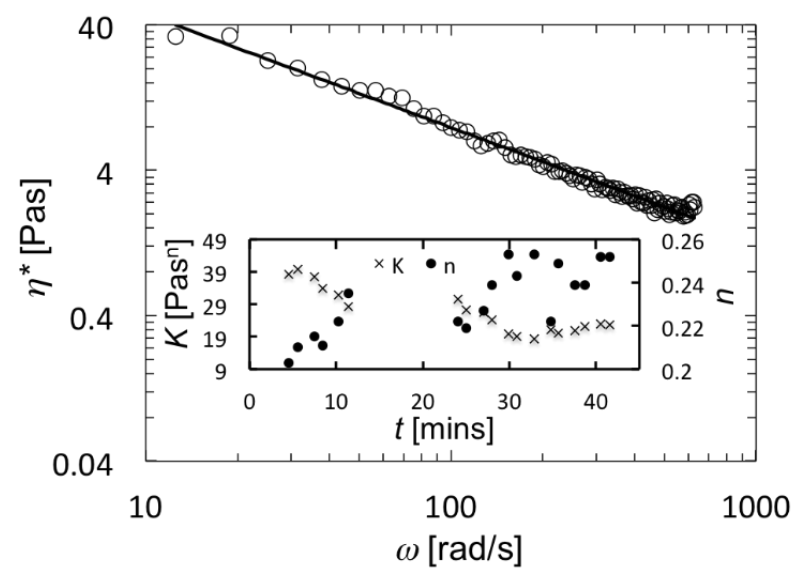

(b)

Figure 2 The inset shows the variation of $K$ and $n$ with time (45 minutes); (a) typical viscoelastic response of thickened tailings; (b) plot of the $\left|\eta^{*}\right|$ as a function of the $\omega$ (symbols) with power-law fit (line)

During this period rheological measurements were collected continuously. In-line density measurements that were also conducted separately during the period were compared to the measurements of the OLR. For the purpose of comparison with the density measurements OLR data at $20 \mathrm{~Hz}(\omega=120.6 \mathrm{rad} / \mathrm{s}), 50 \mathrm{~Hz}$ $(\omega=314.1 \mathrm{rad} / \mathrm{s})$ and $80 \mathrm{~Hz}(\omega=502.6 \mathrm{rad} / \mathrm{s})$ were considered at every time point. The results are shown in Figure 3(a). The filled symbols in the figure represent the density measurements and the unfilled symbols are the complex viscosities measured at $20 \mathrm{~Hz}$ (circles), $50 \mathrm{~Hz}$ (squares) and $80 \mathrm{~Hz}$ (triangles), measured over a period of forty-five minutes. In Figure 3(b) we plot the density as a function of the viscosity at each frequency. It can be observed from Figure 3(b) that the complex viscosity measurements correlate well with the density variations over the range observed in the mine site in the time interval of the experiment. It can also be observed from Figure 3(b) that viscosity at $20 \mathrm{~Hz}$ varies most with the changes in density. We have therefore selected the $20 \mathrm{~Hz}$ signal to compare with the density reading.

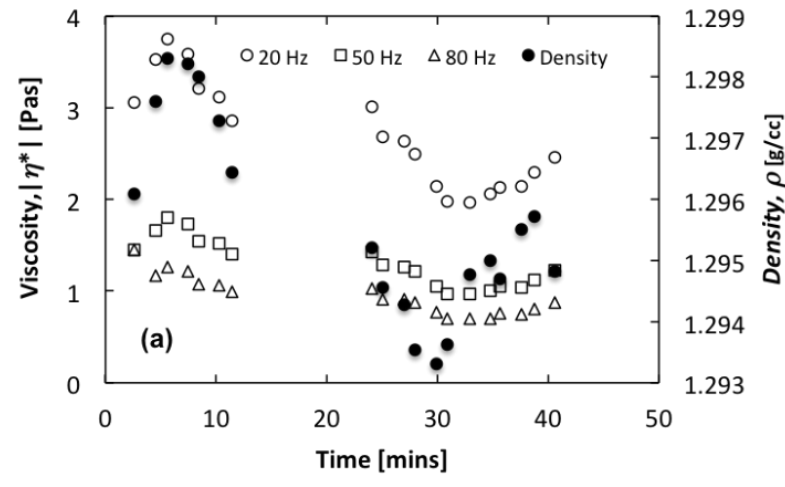

(a)

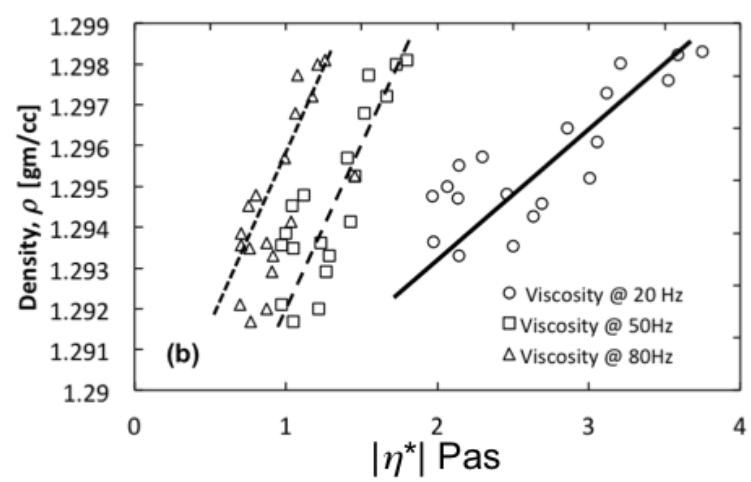

(b)

Figure 3 (a) variation of the $\left|\eta^{*}\right|$ time at three frequencies $(20 \mathrm{~Hz}, 50 \mathrm{~Hz}$, and $80 \mathrm{~Hz})$ compared with the measured underflow density; (b) $\rho$ plotted against $\left|\eta^{*}\right|$ for the three separate frequencies show strong correlation

In Figure 4 we compare the density with the viscosity measured at $20 \mathrm{~Hz}$ oscillation frequency over a period of eight hours. The correlation is strong. It is clear from these comparisons that the longer modes in the rheological spectrum are affected by the changes in density, and it might be possible in future to make better comparisons with the density by choosing the frequency for rheological measurements more carefully. 


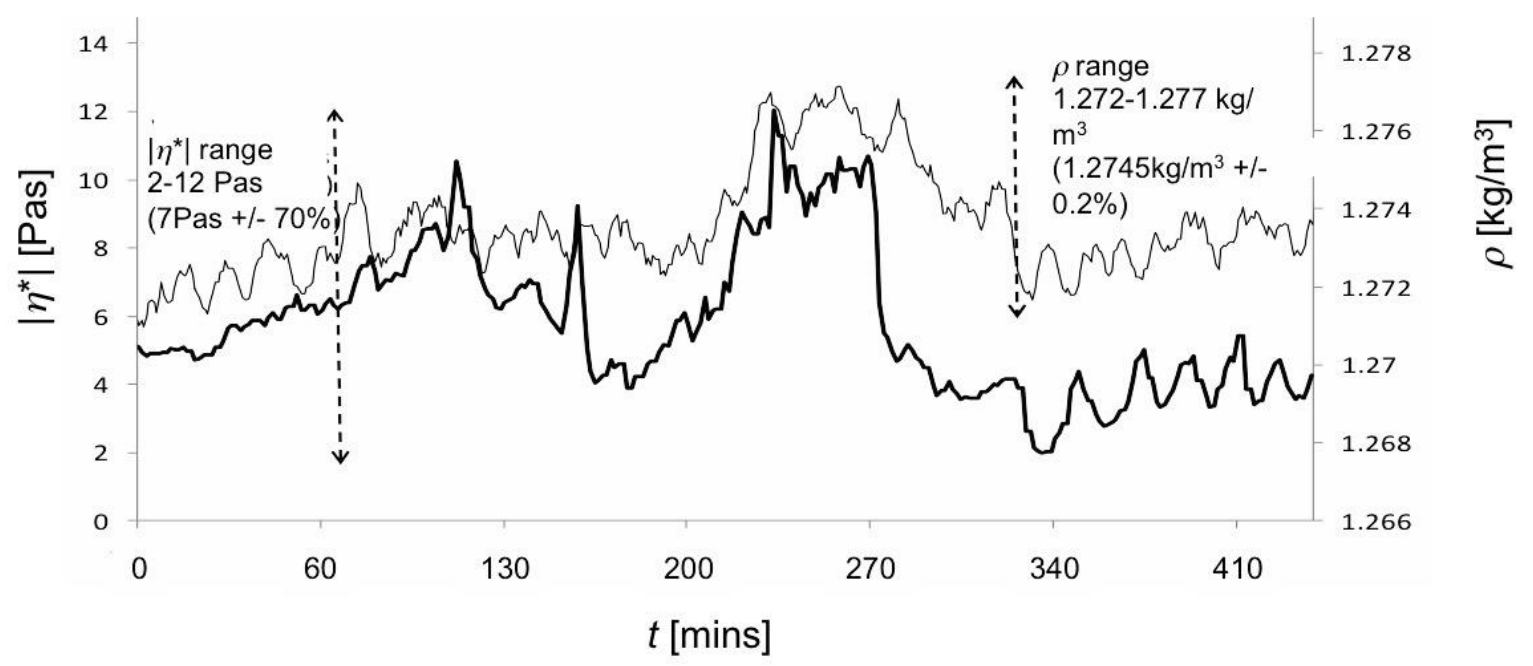

Figure 4 Variation of the underflow density (thin line) and the equivalent in complex viscosity (bold lines) plotted against time for continuous measurements spanning 8 hours

A remarkable feature becomes evident by studying the data in Figure 4 is that despite the variation in the source of the effluent and the relatively strong thickening action expected from the thickener, the measured density varies only about $0.2 \%$ in contrast to about a $70 \%$ variation in the $\left|\eta^{*}\right|$ value measured at $20 \mathrm{~Hz}$. Since the low frequency data greatly influences the magnitudes of $\mathrm{K}$ and $\mathrm{n}$ it is clear, which ultimately determine the flow properties of the tailing, it is clear that relying on the measurements of density alone would be unhelpful because large changes in flow properties can result from seemingly insignificant changes in the measurements of density. Ideally both measurements will be necessary as several of the processing steps would require estimates of all three parameters $\rho, \mathrm{K}$ and $\mathrm{n}$. For instance the standard method (Walker and Goulas, 1984) to estimate the pump Reynolds number $\left(R e_{P}\right)$, for derating the performance of pumps handling non-Newtonian slurries, can be approximated as $R e_{P}=N^{(2-n)} D_{i} \rho / K$, where $\mathrm{N}$ is the revolutions per second of an impeller of diameter $\mathrm{D}_{\mathrm{i}}$. It is obvious that information of $r$ alone is insufficient to enable the evaluation $R e_{P}$ for a given $\mathrm{N}$ and $\mathrm{D}_{\mathrm{i}}$. However with the measurements of the rheological properties described above, it becomes possible for an operator can select the target value of $\left(R e_{P} / D_{i}\right)$, and the rotational speed of the pump motor can be adjusted dynamically depending on the needs of the process. This could provide stable running of the circuits with minimal risk to the capital assets. This will also ensure that the discharge characteristic remain consistently at levels that are stipulated by design or legislation.

\section{Conclusions}

The work reports the application of a novel online rheometer OLR Series 1000 (OLR) developed by Rheology Solutions Pty Ltd for the measurements of the flow properties of coal tailings with a focus of assessing the possibility of using the OLR for process control. It was found that the complex viscosity measured by the OLR correlates well with the density of tailings and that the correlation is more apparent at low frequencies. The correlation was however non-linear and even small changes in density could result in large changes in the flow properties of the material. It is concluded that both measurements would be necessary for effective process control, and an example of how the combined measurements can be used is discussed.

\section{References}

Bell, D., Binding, D. and Walters, K. 2006, 'The oscillatory squeeze flow rheometer: comprehensive theory and a new experimental facility', Rheologica Acta, vol. 46, no. 1, pp. 111-121.

Bird, R. B., Armstrong, A. and Hassager, O. 1977, 'Dynamics of polymeric liquids', Wiley, New York. 
Blight, G.E. and Bentel, G.M. 1983, 'The behaviour of mine tailings during hydraulic deposition', Journal of the South African Institute of Mining and Metallurgy, vol. 83, no. 4, pp. 73-86.

Clayton, S., Grice, T. and Boger, D. 2003, 'Analysis of the slump test for on-site yield stress measurement of mineral suspensions', International Journal of Mineral Processing, vol. 70, no. 1, pp. 3-21.

Engmann, J., Servais, C. and Burbidge, A.S. 2005, 'Squeeze flow theory and applications to rheometry: a review', Journal of nonNewtonian Fluid Mechanics, vol. 132, no. 1, pp. 1-27.

Gawu, S.K. and Fourie, A. 2004, 'Assessment of the modified slump test as a measure of the yield stress of high-density thickened tailings', Canadian Geotechnical Journal, vol. 41, no. 1, pp. 39-47.

Kempton, H., Bloomfield, T.A., Hanson, J.L. and Limerick, P. 2010, 'Policy guidance for identifying and effectively managing perpetual environmental impacts from new hardrock mines', Environmental Science and Policy, vol. 13, no. 6, pp. 558-566.

Konigsberg, D., Nicholson, T., Halley, P., Kealy, T. and Bhattacharjee, P. 2013. 'Online process rheometry using oscillatory squeeze flow', Applied Rheology, vol. 23., no. 3, pp. 35688

Konigsberg, D., Nicholson, T.M., Halley, P.J. and Ahn, K.H. 2014, 'Technical note: correcting for shear strain in an oscillatory squeeze flow rheometer', Rheologica Acta, vol. 53, no. 2, pp. 103-107.

Leblanc, M., Morales, J.A., Borrego, J. and Elbaz-Poulichet 2000, 'A 4500 years old mining pollution in Southwestern Spain: Long-Term concerns for modern mining', Economic Geology, vol. 95, pp. 655-672.

Pashias, N., Boger, D., Summers, J. and Glenister, D. 1996, 'A fifty cent rheometer for yield stress measurement', Journal of Rheology, vol. 40, no. 6, pp. 1179-1189.

Phan-Thien, N. 1980. 'Small strain oscillatory squeeze film flow of simple fluids', Journal of the Australian Mathematical Society. Series B. Applied Mathematics, vol. 22, no. 1, pp. 22-27.

Phan-Thien, N., Nasseri, S. and Bilston, L.E. 2000, 'Oscillatory squeezing flow of a biological material', Rheologica Acta, vol. 39, no. 4, pp. 409-417.

Walker, C.I. and Goulas, A. 1984, 'Performance characteristics of centrifugal pumps when handling non-Newtonian homogenous slurries', Proceedings of the Institution of Mechanical Engineers, vol. 198A, pp. 41-49.

Wingstrand, S.L., Alvarez, N.J., Hassager, O. and Dealy, J.M. 2016, 'Oscillatory squeeze flow for the study of linear viscoelastic behavior', Journal of Rheology, vol. 60, no. 3, pp. 407-418. 